See discussions, stats, and author profiles for this publication at: https://www.researchgate.net/publication/328317127

Monitoring Home-Based Activity of Stroke Patients: A Digital Solution for Visuo-Spatial Neglect Evaluation: Proceedings of the 4th International Conference on NeuroRehabilitation (...

Chapter · January 2019

DOI: 10.1007/978-3-030-01845-0_139

CITATION

1

6 authors, including:

Silvana Giovanna Dellepiane

Università degli Studi di Genova

158 PUBLICATIONS 1,632 CITATIONS

SEE PROFILE
READS

84

Drancesca Cecchi

University of Florence

9 PUBLICATIONS 74 CITATIONS

SEE PROFILE

Some of the authors of this publication are also working on these related projects:

Rehab@Home View project

Project image and volume segmentation View project 


\title{
Monitoring Home-Based Activity of Stroke Patients: A Digital Solution for Visuo-Spatial Neglect Evaluation
}

\author{
M. Morando, E. Bacci Bonotti, G. Giannarelli, S. Olivieri, S. Dellepiane, F. Cecchi
}

\begin{abstract}
The possibility to prescribe home-based rehabilitation activity after stroke strongly increases the amount of exercises to perform, thus helping the maintenance of relearned skills, the completion of the rehabilitation program, the practice of physical and mental concentration. Even more important is the monitoring of the patient activity at home, as it is provided by the Remote Monitoring Validation Engineering System (ReMoVES) platform [1]. The present work refers to the implementation and integration in ReMoVES platform of a digital and web-based version of Albert's [2] and Line Bisection [3] tests devoted to visuo-spatial neglect evaluation and its remote monitoring. A statistical analysis devoted to validating testretest reliability is proposed. Concurrent correlation between digital and traditional administration of the tests is presented, in order to evaluate the validity of the remote monitoring of the home-administration through ReMoVES platform.
\end{abstract}

\section{INTRODUCTION}

Patients suffering from the neuropsychological syndrome of unilateral spatial neglect (USN) fail to orient, report, or respond to stimuli in contralesional space after a cerebral stroke. Since it is not caused by primary sensory or motor dysfunction, USN is considered a higher-order disorder [4]. Specific neuro-rehabilitation is then required, helping the reacquisition of the functional ability and a better quality of life, along with appropriate tests to evaluate the disorder recovery.

To this end, ReMoVES platform has been equipped with cloud data-acquisition capabilities to allow the therapist to remote monitoring the patient activity and progress. In addition to specific exercises proposed for the training of visual attention in the neglected region, appropriate tests to continuously evaluate the visuo-spatial disorder severity have been added.

The present work refers to the implementation and integration in ReMoVES platform of a digital and web-based version of Albert's [2] and Line Bisection [3] tests devoted to visuo-spatial neglect evaluation and its remote monitoring. The tests have been implemented by following the traditional paper-based test version. The validity of the approach is based on a statistical study where the parameters usually extracted by the therapist attending the test are correlated with the parameters automatically extracted from the webbased version. In the following, the implemented test version is described, along with the data and key indicators which allow the monitoring of the actual patient performance.

F. Cecchi, E. Bacci Bonotti, G. Giannarelli and S. Olivieri are from Fondazione Don Carlo Gnocchi (fcecchi@dongnocchi.it).

S. Dellepiane and M. Morando are from DITEN, Università degli Studi di Genova (morando.matteo@gmail.com, silvana.dellepiane@unige.it).

\section{METHODS}

ReMoVES platform has been developed by Department of Naval, Electrical, Electronics, and Telecommunications Engineering (DITEN) of Universitá degli Studi di Genova, as a platform that addresses the problem of continuity of care in a smart and cost-effective way [1].

Two brand-new serious games have been developed and have been included in the ReMoVES platform. These activities are the digital version of paper-and-pencil tasks that are commonly applied in mapping visuo-spatial neglect in clinical practice. A similar solution have been already deployed in [5]. We introduce novelty thanks to the ReMoVES platform: the patients can run the task repeatedly at their home, without the clinician supervision.

\section{A. Digital Albert's test}

In this test, patients must cross out forty $2.5 \mathrm{~cm}$ lines that are placed in pseudo-random orientations on a piece of paper, using a pencil. The actual disposition of these lines is standardised, allowing for a systematic analysis of subjects' performance on the left, the right, and the centre of the page. Scoring is based on the number and location of lines left uncrossed [2]. In the digital version the patient interacts with a touchscreen (Fig. 1a). Invalid and accidental touches are automatically ignored by the system. The paper-based and the digital test differ on these aspects:

- patient must be capable to hold a pencil / patient must be able to use a touch screen;

- the digital version can provide more granular information on the speed execution and the cross out order.

\section{B. Digital Line Bisection test}

To complete the test, one must place a mark with a pencil through the centre of a series of horizontal lines. The test is scored by measuring the deviation of the bisection from the true centre of the line [3]. In the digital version the patient interacts with a touchscreen (Fig. 1b). The paper-based and the digital test differ on these aspects:

- patient must be capable to hold a pencil / patient must be able to use a touchscreen;

- the digital version allows customisation of the colour, width, height, position and rotation of the line in order to provide multiple variants of the standard test and indepth analysis;

- the digital version displays a new line whenever the patient manages to cross out the line, continuously up to two minutes. This allows repeating the measurements 


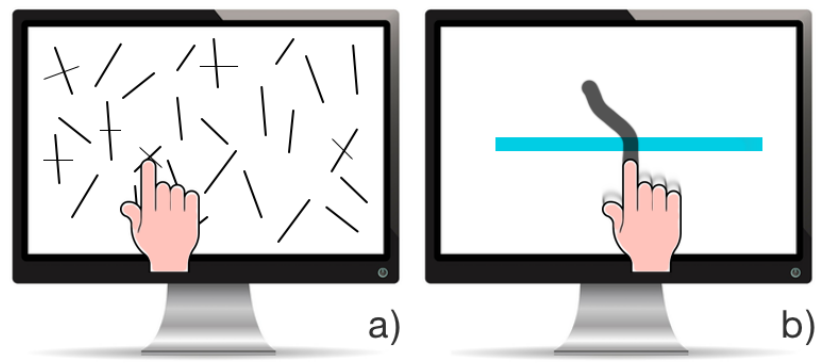

Fig. 1. a) Digital and web-based version of Albert's test included in ReMoVES platform b) Digital and web-based version of Line Bisection test included in ReMoVES platform

more times than the traditional test that is based on just three lines.

\section{Statistical study}

We are providing preliminary results. We want to offer an overall description of the method that has been tested with 10 subjects so far.

[6] reported an excellent test-retest reliability for the Line Bisection test $(\rho=0.73)$ and for the Albert's test ( $\rho=0.79$ ). More recently, [7] examined the test-retest reliability of the Line Bisection test in elderly patients with stroke who repeated the test within the hour. The intraclass correlation coefficient (ICC) was excellent for patients with neglect $(\mathrm{ICC}=0.97)$.

In [8] an excellent correlation of Albert's test with the Line Bisection test $(\rho=0.85)$ was found when comparing the performance of 57 elderly patients with stroke.

[9] examined a computer-based method of administering the Albert's test in patients with neglect, without neglect, and age-matched control subjects. Significant differences were found between subjects with neglect and those without neglect, as well as subjects with neglect and age-matched controls.

Similarly, the two digital versions implemented in this paper are at present under validation for test-retest reliability and for investigating convergent correlation. As proposed in [10], test-retest reliability (or ICC) is considered excellent with values larger than 0.75 ; correlation values larger than 0.6 are considered excellent in convergence analysis.

In our clinical trials, the subjects repeated the Line $\mathrm{Bi}$ section test and the Albert's test twice; we collected these indicators and these test-retest results:

- Line Bisection test

- Offset: mean of the measured deviations from the centre of the lines during the whole session. Resulting Pearson correlation coefficient is $\rho=0.83$

- Rate: how many lines the patient manages to cross out every second. Resulting Pearson correlation coefficient is $\rho=0.94$

\section{- Albert's test}

- Uncrossed: count of the lines left uncrossed on the same side of the touchscreen. Resulting Pearson correlation coefficient is respectively $\rho=0.98$ and $\rho=0.80$ for left and right sides;

- Execution order: left to right, right to left or undefined crossing out pattern followed by the patient. Six out of the ten patients kept the same strategy: the resulting ratio is $r=0.6$.

Finally, concurrent evaluation of digital and traditional administration of the tests has been addressed, in order to assess the feasibility of the remote monitoring of the homeadministration through ReMoVES platform. For the subject \#1 -affected by a rare right-sided visuo-spatial neglect- both digital tests detect the presence of this syndrome while the traditional test didn't provide relevant results. For subject \#9 conventional and digital Albert's tests produced nearly identical results, highlighting left hemispatial neglect; likewise, the patient showed difficulty and confusion during the execution of Line Bisection Test in both modes. For subject \#10 a significant correlation between traditional and digital Line Bisection tests emerges. The tests administered to other subjects did not provide significant results in either electronic or paper form.

\section{CONCLUSION}

By the implemented automated test, it is easy to prove that the disease severity of a visual USN can be assessed even for de-hospitalised patients, without the need of their transfer to the clinical ambulatory. The developed assessment approach is also proposed for a further correlation with parameters extracted by the ReMoVES games. In such a way, it will be possible to evaluate whether repetitions of physical and cognitive exercises at home favour the maintenance of intensive rehabilitation program results acquired during the hospitalization period.

\section{REFERENCES}

[1] M. Morando, S. Ponte, E. Ferrara, and S. Dellepiane, "Definition of motion and biophysical indicators for home-based rehabilitation through serious games," Information, vol. 9, no. 5, 2018.

[2] M. L. Albert, "A simple test of visual neglect.," Neurology, 1973.

[3] "Stroke Engine Line Bisection test." https://www.strokengine.ca. Accessed: 2018-05-18.

[4] K. M. Heilman, E. Valenstein, and R. T. Watson, "Neglect and related disorders," in Seminars in neurology, vol. 20, pp. 463-470, Copyright(C) 2000 by Thieme Medical Publishers, Inc., 333 Seventh Avenue, New York, NY 10001, USA. Tel.:+ 1 (212) 584-4662, 2000.

[5] N. Vaes, C. Lafosse, G. Nys, H. Schevernels, L. Dereymaeker, K. Oostra, D. Hemelsoet, and G. Vingerhoets, "Capturing peripersonal spatial neglect: An electronic method to quantify visuospatial processes," Behavior research methods, vol. 47, no. 1, pp. 27-44, 2015.

[6] M.-J. C. Sea and A. Henderson, "The reliability and validity of visuospatial inattention tests with stroke patients," Occupational Therapy International, vol. 1, no. 1, pp. 36-48, 1994.

[7] M. J. Bailey, M. J. Riddoch, and P. Crome, "Test-retest stability of three tests for unilateral visual neglect in patients with stroke: Star cancellation, line bisection, and the baking tray task," Neuropsychological Rehabilitation, vol. 14, no. 4, pp. 403-419, 2004.

[8] B. M. AGRELL, O. I. DEHLIN, and C. J. DAHLGREN, "Neglect in elderly stroke patients: a comparison of five tests," Psychiatry and clinical neurosciences, vol. 51, no. 5, pp. 295-300, 1997.

[9] J. Potter, T. Deighton, M. Patel, M. Fairhurst, R. Guest, and N. Donnelly, "Computer recording of standard tests of visual neglect in stroke patients," Clinical Rehabilitation, vol. 14, no. 4, pp. 441-446, 2000.

[10] "Stroke Engine Albert's test." https://www.strokengine.ca/. Accessed: 2018-05-18. 
TABLE I

LINE BISECTION TEST: OFFSET

\begin{tabular}{|l|c|c|c|c|c|c|c|c|c|c|}
\hline & Sub. 1 & Sub. 2 & Sub. 3 & Sub. 4 & Sub. 5 & Sub. 6 & Sub. 7 & Sub. 8 & Sub. 9 & Sub. 10 \\
\hline Test & $-22.3 \%$ & $+2.7 \%$ & $+4.6 \%$ & $-0.5 \%$ & $-5.0 \%$ & $+5.1 \%$ & $+10.0 \%$ & $-1.5 \%$ & $+2.1 \%$ & $+9.1 \%$ \\
\hline Retest & $-24.0 \%$ & $+6.4 \%$ & $+6.8 \%$ & $+3.3 \%$ & $-6.5 \%$ & $+2.0 \%$ & $-4.1 \%$ & $-0.7 \%$ & $-4.1 \%$ & $+13.2 \%$ \\
\hline
\end{tabular}

The mean offset from the centre of the line is provided as percentage. Negative values are offset to the left. Test-retest correlation is: $\rho=0.83$

TABLE II

LINE BISECTION TEST: RATE

\begin{tabular}{|l|r|r|r|r|r|r|r|r|r|r|}
\hline & \multicolumn{1}{|r|}{ Sub. 1 } & \multicolumn{1}{|c|}{ Sub. 2 } & \multicolumn{1}{|c|}{ Sub. 3 } & \multicolumn{1}{|c|}{ Sub. 4 } & Sub. 5 & Sub. 6 & Sub. 7 & Sub. 8 & Sub. 9 & Sub. 10 \\
\hline Test & 0.56 & 0.35 & 0.37 & 0.82 & 0.76 & 0.30 & 0.74 & 1.27 & 0.16 & 0.62 \\
\hline Retest & 0.42 & 0.51 & 0.34 & 1.15 & 0.94 & 0.35 & 0.66 & 1.37 & 0.10 & 0.57 \\
\hline
\end{tabular}

The bisection rate is provided as lines crossed every second. Test-retest correlation is: $\rho=0.94$

TABLE III

ALBERT'S TEST: UNCROSSED ON THE LEFT

\begin{tabular}{|l|c|c|c|c|c|c|c|c|c|c|}
\hline & Sub. 1 & Sub. 2 & Sub. 3 & Sub. 4 & Sub. 5 & Sub. 6 & Sub. 7 & Sub. 8 & Sub. 9 & Sub. 10 \\
\hline Test & 1 & 0 & 0 & 3 & 3 & 0 & 1 & 4 & 17 & 0 \\
\hline Retest & 0 & 0 & 0 & 5 & 6 & 0 & 2 & 4 & 17 & 0 \\
\hline
\end{tabular}

Number of lines uncrossed on the left side of the screen. $\rho=0.98$

TABLE IV

ALBERT'S TEST: UNCROSSED ON THE RIGHT

\begin{tabular}{|l|c|c|c|c|c|c|c|c|c|c|}
\hline & Sub. 1 & Sub. 2 & Sub. 3 & Sub. 4 & Sub. 5 & Sub. 6 & Sub. 7 & Sub. 8 & Sub. 9 & Sub. 10 \\
\hline Test & 3 & 0 & 0 & 5 & 3 & 0 & 1 & 4 & 6 & 1 \\
\hline Retest & 0 & 0 & 0 & 3 & 5 & 0 & 1 & 3 & 5 & 0 \\
\hline
\end{tabular}

Number of lines uncrossed on the right side of the screen. $\rho=0.80$

TABLE V

ALBERT'S TEST: PATTERN

\begin{tabular}{|l|c|c|c|c|c|c|c|c|c|c|}
\hline & Sub. 1 & Sub. 2 & Sub. 3 & Sub. 4 & Sub. 5 & Sub. 6 & Sub. 7 & Sub. 8 & Sub. 9 & Sub. 10 \\
\hline Test & L & M & R & M & L & L & M & M & M & R \\
\hline Retest & L & M & L & M & R & M & L & M & M & R \\
\hline
\end{tabular}

Crossing out pattern: $\mathrm{R}$ is from right to left, $\mathrm{L}$ is from left to right, $\mathrm{M}$ is from middle or not a defined pattern. $r=0.6$

TABLE VI

RELEVANT RESULTS SUMMARY

\begin{tabular}{|c|c|c|c|c|c|c|c|c|c|c|}
\hline & Sub. 1 & Sub. 2 & Sub. 3 & Sub. 4 & Sub. 5 & Sub. 6 & Sub. 7 & Sub. 8 & Sub. 9 & Sub. 10 \\
\hline Diagnosis & $\begin{array}{c}\text { Right } \\
\text { neglect }\end{array}$ & $\begin{array}{c}\text { Post-stroke } \\
\text { no neglect }\end{array}$ & & & & & $\begin{array}{c}\text { Post-stroke } \\
\text { no neglect }\end{array}$ & & $\begin{array}{c}\text { Left } \\
\text { neglect }\end{array}$ & $\begin{array}{c}\text { Left } \\
\text { neglect }\end{array}$ \\
\hline Trad. Albert & & & & & & & & & $16 \mathrm{~L}-1 \mathrm{R}$ & \\
\hline Trad. Bisection & & & & & & & & & confused & $+10.0 \%$ \\
\hline Digit. Bisection & $-23.6 \%$ & & & & & & & & $\begin{array}{l}\text { confused } \\
\text { and slow }\end{array}$ & $+11.2 \%$ \\
\hline
\end{tabular}

Small differences between left and right in Albert's test and percentages in $-10 \% / 10 \%$ range in Line Bisection test offset are omitted (empty cells). 